\title{
Programa de reinserción social para fortalecer el modelo de gestión penitenciaria en las personas privadas de libertad en la ciudad de Guayaquil, Ecuador 2020
}

\author{
Abel Rolando Jiménez Vargas \\ ababeljimenezv2011@ hotmail.com \\ Escuela de Posgrado: Programa Académico \\ Doctorado en Gestión Pública y Gobernabilidad \\ Universidad de Guayaquil. \\ Piura-Perú
}

\section{RESUMEN}

El presente trabajo de investigación abordó el tema del Modelo de Gestión Penitenciaria, situación que viene generando una inadecuada política penitenciaria la misma que no permite generar una pertinente reinserción por parte de las personas privadas de su libertad, y tuvo como objetivo general proponer un programa de reinserción social para fortalecer el modelo de gestión penitenciaria en las personas privadas de libertad en la ciudad de Guayaquil. La metodología tuvo un propósito como Aplicado, enfoque mixto y un alcance Descriptivo y Explicativo, donde el diseño fue no experimental de corte transversal, se obtuvo una muestra de 100 abogados que laboran atendiendo casos en los establecimientos penitenciarios de la ciudad de Guayaquil. Los resultados mostraron de como los talleres psicopedagógicos impulsan la prevención delictiva. Fortalecen programas de emprendimientos, oportunidades laborales como parte de un programa de reinserción, asimismo, se obtuvo que no se está manejando correctamente la incorporación de los nuevos reclusos. Concluyendo que un Programa de Reinserción Social fortalece un Modelo de Gestión Penitenciaria, y que está de acuerdo con la opinión de especialistas y sobre el análisis que arrojaron un Coeficiente de validez de contenido es 0.908 se recomienda la pertinencia de implementar y aplicar el programa.

Palabras Clave: modelo de gestión penitenciaria; programa de reinserción social; personas privadas de su libertad; readaptación; oportunidad laboral. 


\title{
Social reintegration program to strengthen the penitentiary management model in persons deprived of liberty in the city of Guayaquil, Ecuador. 2020
}

\begin{abstract}
The present research work addressed the subject of the Penitentiary Management Model, a situation that has been generating an inadequate prison policy, which does not allow the generation of a pertinent reintegration on the part of persons deprived of their liberty, and its general objective was to propose a program of social reintegration to strengthen the prison management model for people deprived of liberty in the city of Guayaquil. The methodology had a purpose such as Applied, mixed approach and a Descriptive and Explanatory scope, where the design was non-experimental, cross-sectional, a sample of 100 lawyers who work attending cases in the prisons of the city of Guayaquil was obtained. The results showed how psychopedagogical workshops promote crime prevention. They strengthen entrepreneurship programs, job opportunities as part of a reintegration program, and it was also found that the incorporation of new inmates is not being handled correctly. Concluding that a Social Reintegration Program strengthens a Penitentiary Management Model, and that it agrees with the opinion of specialists and on the analysis that yielded a content validity coefficient of 0.908 , the relevance of implementing and applying the program is recommended.
\end{abstract}

KeyWords: prison management model; social reintegration program; people deprived of their liberty; rehabilitation; job opportunity.

Artículo recibido: 10 Agosto. 2021 Aceptado para publicación: 07. Setiembre. 2021 Correspondencia: ababeljimenezv2011@ hotmail.com

Conflictos de Interés: Ninguna que declarar 


\section{INTRODUCCIÓN}

A nivel mundial existen retos muy importantes a romper y entre ellos tenemos el de los centros penitenciarios, este reto se llama: La rehabilitación y la reintegración, que buscan mejorar la capacidad de los Estados por gestionar eficazmente la Gestión de Reclusos en las prisiones, se centra especialmente en un número selecto de países de Asia, el Norte de África y el Oriente Medio, entre ellos Kazajstán, Uganda y Túnez. El proyecto prevé el inicio de programas de desvinculación en las prisiones que está orientado a disuadir extremistas de la violencia y establecer normas que faciliten su reintegración social después de recuperar la libertad. El proyecto está dirigido a las administraciones nacionales penitenciarias, reclusos extremistas violentos y la población carcelaria en general, así como a las organizaciones de la sociedad civil que participan en la separación y la reintegración social de antiguos extremistas violentos. (Voronkov, 2019, párr. 1-2)

Según el informe de Bordignon (2019) en una entrevista realizada por el Magazine JUSTICE TRENDS, manifiesta que uno de los principales proyectos es tratar de maximizar la disponibilidad de plazas. Tenemos varias acciones y una de ellas es la de aumentar las obras penitenciarias. Abrir plazas no solo significa construir unidades penitenciarias. Necesitamos y estamos invirtiendo en una política de seguimiento electrónico. Hoy en día, Brasil tiene más de 50000 personas que utilizan tobilleras electrónicas. Es una alternativa al encarcelamiento, tanto para las sentencias cautelares como para el régimen semiabierto. En algunos casos, el recluso lleva la tobillera y se va a dormir a su propia casa. Esto evita la necesidad de mayores inversiones en unidades penitenciarias. Tenemos un déficit de plazas. También es necesario involucrar a la iniciativa privada en la construcción de nuevas unidades penitenciarias a través de alianzas público-privadas y de la cogestión con el sector público y privado. Hay varias acciones: apertura de plazas, restauración del control y el trabajo también muy importante de la inteligencia penitenciaria. (párr. 7-9)

La UNODC (2015), partiendo del apuro de capacitar a los miembros de su entidad, elaboro en el marco del plan SECOPA, un proyecto estructurado por la Oficina Regional de las Naciones Unidas contra la Droga y el Delito para Centroamérica y el Caribe en Panamá (UNODC ROPAN), con el financiamiento de la Unión Europea, dicho proyecto "Derechos Humanos y Gestión Penitenciaria" estuvo aplicado al personal de seguridad en los centros penitenciarios de la zona metropolitana. (párr. 1)

Según Aquino (2016) en contexto de la otorgación del equipamiento al personal de 
seguridad, anunció que seis meses tardaría la reconfiguración del sistema penitenciario queretano, donde se privilegiará el cambio de paradigma y apoyará a la reinserción social a través de esquemas laborales, esta reingeniería, incluirá un paquete de reformas para armonizar la Ley Nacional de Ejecución de Sanciones, para contar con un sistema penitenciario con visión humanista y enfrentar la problemática de inseguridad en el estado. El funcionario de Estado comentó que se trabajará en cinco ejes: rediseño de la infraestructura física, fortalecimiento de la tecnología que se sumará al eje de Coordinación, Comando, Control, enlaces de comunicación y equipos de cómputo (C5), creación de esquemas laborales para los reos, perfeccionar la administración de los centros penitenciarios y la selectividad en el proceso de reclutamiento del personal. Para llegar al resultado de esta capacitación, el funcionario sostuvo, que el personal del centro de reinserción juvenil se encuentra en evaluación, a fin de conseguir la certificación internacional de la Embajada de los Estados Unidos, y de la Asociación de Certificación de Prisiones. (párr. 1-5)

Es importante considerar que además del problema de la violencia se suma a lo que hoy se vive a nivel mundial, es la Pandemia COVID 19, así como, otros problemas estructurales del país, esto debido al hacinamiento y a las condiciones precarias que viven los centros penitenciarios en diferentes partes del mundo. En el Perú, en este marco, el Tribunal Constitucional ha emitido una sentencia (expediente $\mathrm{N}^{\circ} 05436-2014-\mathrm{PHC} / \mathrm{TC}$ ) donde declara el "estado de cosas inconstitucional" de las cárceles en Perú, debido a la aglomeración existente en las mismas. En este decreto ordenado por el poder ejecutivo, se propone desarrollar una estrategia para desarrollar esta problemática hasta el año 2025, si hasta ese entonces no se logra, se procederá a cerrar las cárceles al hacinamiento que existe. En esta resolución, ordena al poder ejecutivo (especialmente al Minjus) elaborar un plan para solucionar el problema hasta 2025. Si no lo hace para entonces, cerrará las cárceles. De esta manera, en este análisis se realizan sugerencias sobre los tópicos donde se debería enfocar en este artículo se hacen algunas sugerencias sobre los puntos donde debería enfocarse esta estrategia. (párr. 1-5)

Con estas evidencias que se pueden ver en el día a día, es que nos preguntamos de ¿Cómo el estado puede brindar soluciones a este tipo de problemas con centros penitenciarios con demasiadas limitantes?, esto nos lleva a reflexionar y plantear la siguiente pregunta de investigación consistente en ¿Cómo fortalecer el modelo de gestión penitenciaria en las 
personas privadas de libertad en la ciudad de Guayaquil, Ecuador? 2020?, esta problemática, se ha justificado teniendo en cuenta que el estado debe priorizar acciones de control en los centros penitenciarios con la finalidad de poder reintegrar a la sociedad a aquellas personas que terminaron una condena y que al mismo tiempo fueron preparados y orientados a reinsertarse en la sociedad, pero que a su vez necesita fortalecer su accionar regulado durante todo ese proceso de formación en el cual intervienen muchos profesionales, pero que al mismo tiempo son insuficientes. Es en ese sentido, que podemos justificar la presente investigación en lo Teórico, aduciendo que el conocimiento no solo es recibir información y retenerla, sino más por el contrario hacer que el conocimiento pueda cumplir su ciclo a través del cual la información del conocimiento quede plasmada en cada individuo, con ello se estaría logrando mostrar un conocimiento existente pero que puede ser ampliado con esta investigación y que a su vez tributará a nuevas investigaciones que planteen temáticas parecidas o similares. Asimismo, justifica en lo práctico porque el cúmulo de conocimientos se podrán aplicar en la resolución de problemas que se presentan en los diferentes entornos de la vida, cuando el conocimiento toma posición frente a los problemas, invita los profesionales a tomar las acciones correspondientes en pos de lograr estratégicamente una solución viable y oportuna, y en lo social, esta postura conlleva a la sociedad a comprender que con el nuevo conocimiento o el ampliado o actualizado, se contribuye a lograr soluciones prácticas que contribuye a la mejora en la calidad de vida de los moradores y este caso a la atención recuperativa de las personas privadas de su libertad, indistintamente de la razón por la cual llegaron.

\section{Esto nos permite plantear como}

\section{Objetivo General}

- Proponer un programa de reinserción social para fortalecer el modelo de gestión penitenciaria en las personas privadas de libertad en la ciudad de Guayaquil, Ecuador. $2020 "$.

\section{Teniendo además como}

\section{Objetivos específicos}

1. Diagnosticar el estado actual del modelo de gestión penitenciaria en las personas privadas de libertad en la ciudad de Guayaquil,

2. Identificar los factores influyentes en el modelo de gestión penitenciaria en las 
personas privadas de libertad en la ciudad de Guayaquil,

3. Diseñar programa de reinserción social para fortalecer el modelo de gestión penitenciaria para las personas privadas de libertad en la ciudad de Guayaquil.

4. Estimar los resultados que generará la implementación de programa de reinserción social en el modelo de gestión penitenciaria en las personas privadas de libertaden la ciudad de Guayaquil.

\section{Esto ha permitido platear como}

\section{Hipótesis}

- La implementación de un programa de reinserción social fortalecería el modelo de gestión penitenciaria en las personas privadas de libertad en la ciudad de Guayaquil, Ecuador. 2020.

\section{METODOLOGÍA.}

\subsection{Tipo y diseño de investigación.}

Según el Propósito de Tipo Aplicada, ya se caracteriza por buscar la aplicación de estos conocimientos y su utilización a la realidad, se requiere de un marco teórico para resolver necesidades y problemas sociales. Según el enfoque es mixta ya que se combinó la investigación cuantitativa y cualitativa y utilizando las fortalezas de las dos, armonizándolos es decir combinándolos. Hernández-Sampieri, Mendoza, 2018, (p. 610). Según el alcance es Descriptiva, porque define las características propias de una realidad en particular, sin que medie condición alguna para indagar que cosas son las que la motivan. (Técnicas de Investigación, 2020) y es Explicativa, porque busca establecer la relación que producirá un efecto nuevo o esperado al sobre hechos puntuales están direccionadas exponer las causas de los eventos. (Dempwolff, 2013). Asimismo, es transeccional-causal, no experimental, fundamentado en que recolectaremos datos y vamos a describir variables para analizarlos y conocer su incidencia e interrelación; de corte transversal puesto que vamos a realizar una mediación de nuestras variables. Sampieri (s. f.) (p.610).

\subsection{Variables y operacionalización.}

\section{- Variable Independiente: Programa de Reinserción Social}

De acuerdo con Ministerio de Justicia (2018) la reinserción social es un proceso sistemático que implica desarrollar acciones orientadas a favorecer la integración de una persona privada de su libertad por infringir la ley a la sociedad. (párr. 1), y 


\section{- Variable Dependiente: Modelo de Gestión Penitenciaria}

De acuerdo con Morales (2016) la gestión penitenciaria debe entenderse como la gestión de las personas que tienen a cargo la administración penitenciaria, así mejorar la gestión penitenciaria es mejorar la administración como organización y el proceso penitenciario.

\subsection{Población, muestra y muestreo.}

\section{Población}

Según Sampieri (s. f.) es el universo de personas las mismas que coinciden en las características similares motivo de nuestro estudio que se va a realizar un estudio en un tiempo concluyente. (p. 174). En la presente investigación objeto de estudio se ha considerado como población a los abogados que laboran atendiendo casos en los establecimientos penitenciarios de la ciudad de Guayaquil, la misma que es una cantidad desconocida.

\section{Muestra}

Según Sampieri et al. (2014), lo considera como grupo de elementos, los cuales han sido seleccionados de la población y que la utilizaremos para nuestro estudio en la recolección de datos, para lo cual se utilizara la técnica de muestreo. (p. 175), este muestreo será No probabilístico y utilizaremos el muestreo por conveniencia con la opinión de juristas para que estos tengan un referente, dado que no se conoce el número exacto de abogados participantes.

Por lo tanto se tomará a la una muestra de tamaño $\mathrm{n}=100$

\subsection{Técnicas e Instrumentos de recolección de datos.}

La encuesta de acuerdo con Casas, Repullo y Donado (2002) refieren que esta técnica tiene una frecuencia de uso muy alta en los procesos investigativos, pues permite recabar en forma oportuna y de fácil comprensión la dinámica establecida. Para el presente trabajo se hizo uso de Google Formularios vía online.

Como instrumento se utilizó el cuestionario; la misma que consta de un conjunto de preguntas de acuerdo a nuestras 2 variables motivo de nuestro estudio que tiene que relacionarse con el planteamiento de nuestro problema, dirigido a los abogados descritos en la población.

\subsection{Procedimientos}

Estos estuvieron enmarcados en poder analizar la situación que nos involucraba en la investigación para luego plantear cuales eran los demás pasos a seguir, se recogió 
información válida para la construcción del sustento que respaldara la investigación, seguidamente la aplicación de las encuestas, el análisis de datos y con ello tener los elementos necesarios para evidenciar los resultados de manera concreta.

\subsection{Método de análisis de datos}

En el presente estudio se aplicó la estadística descriptiva en donde describiremos los datos para resumir la información obtenida, para lo cual se desarrolló tablas descriptivas con frecuencias y porcentajes; técnicas graficas mediante la elaboración de figuras dado a conocer en forma organizada y resumida los datos de nuestra muestra, asimismo se utilizó la estadística inferencial con la finalidad de conocer si las variables de investigación guardaban relación y de cuanto era ello.

\subsection{Aspectos éticos}

Acorde con los aspectos deontológicos del código de ética la presente investigación siguió estrictamente los siguientes aspectos: los permisos necesarios de las personas involucradas en brindar las respuestas al cuestionario de recogida de datos, además se explicó sobre la importancia de la confidencialidad de la información vertida, por ello los cuestionarios no preguntaban nombres o apellidos, algo que los identificara. Se buscó además salvaguardar estrictamente la integridad de la información obtenida y esta que no perjudique a otras personas o instituciones teniendo en cuenta la honestidad, la ética, relevancia investigativa.

\section{RESULTADOS Y DISCUSIÓN}

\section{Tabla 1}

Los talleres psicopedagógicos vienen impulsando la prevención delictiva como parte de un programa de reinserción social

\begin{tabular}{ccc}
\hline Descripción & $\mathbf{f i}$ & \% \\
\hline TD & 6 & 6.00 \\
D & 26 & 26.00 \\
NO & 24 & 24.00 \\
A & 44 & 44.00 \\
\hline Total & $\mathbf{1 0 0}$ & $\mathbf{1 0 0 . 0 0}$ \\
\hline
\end{tabular}

\section{Fuente: El Autor}

De acuerdo con los datos obtenidos de la Tabla 1, sobre si Los talleres psicopedagógicos vienen impulsando la prevención delictiva como parte de un programa de reinserción social, al respecto un $6 \%$ manifiestan estar Totalmente en Desacuerdo y un $26 \%$ solo 
refieren estar en Desacuerdo con tal afirmación, asimismo un $24 \%$ no tiene muy en claro tal situación y prefiere No Opinar, mientras que un $44 \%$ ante dicha afirmación refieren estar de Acuerdo. Esto se puede corroborar con lo expuesto por Gilardi (2020) quien manifestó que identifico que como parte del proceso de reinserción social cuando éste es "exitoso"; es decir, el reconocimiento de las emociones desde la recuperación y resignificación de la memoria a través del testimonio. Además, es importante considerar como un espacio de reflexión (llamados talleres) que los conlleve a mejorar conductas y actitudes positivas para una mejor aceptación en la sociedad, desarrollar acciones de espiritualidad o afectividad y, hacia un autoconocimiento en un nivel más consciente. (p. 53), asimismo, Martín-González et al. (2020) refieren que la experiencia en relación con la droga y las formas de reinsertar en la sociedad a los exconvictos expresa que esto debe conlleva el tipo de practica realizada. (p. 381) y Solórzano-Soto, Márquez-Allauca y Márquez-Allauca (2017) manifiesta que el plan de terapia de tipo cognitivo - conductual, determina en gran medida a la rehabilitación y la reinserción social. Este tratamiento, les permite formar parte de un proceso de relajación, control de la ansiedad, promover práctica de deporte, participar de actividades que estimulen la recreación y las relaciones intrapersonales, conlleva generar estímulos satisfactorios de aprendizajes, comunicación y minimizando el decaimiento. (p. 753).

\section{Tabla 2}

Los programas de reinserción social están formando a través de la capacitación a nuevos Emprendedores

\begin{tabular}{ccc}
\hline Descripción & $\mathbf{f i}$ & $\%$ \\
\hline D & 43 & 43.00 \\
NO & 24 & 24.00 \\
A & 15 & 15.00 \\
TA & 18 & 18.00 \\
\hline Total & $\mathbf{1 0 0}$ & $\mathbf{1 0 0 . 0 0}$ \\
\hline
\end{tabular}

Fuente: El Autor

De acuerdo con los datos obtenidos de la Tabla 2, sobre si Los programas de reinserción social están formando a través de la capacitación a nuevos Emprendedores, al respecto un $43 \%$ solo refieren estar en Desacuerdo con tal afirmación, asimismo un $24 \%$ no tiene muy en claro tal situación y prefiere No Opinar, mientras que un $15 \%$ ante dicha 
afirmación refieren estar de Acuerdo y finalmente el $18 \%$ están Totalmente de Acuerdo con lo manifestado. Estos resultados se corroboran con lo evidenciado por Ochoa-García y Martínez- Camacho (2020) manifiestan que el estado de Jalisco le da importancia a la reinserción de los reclusos a la sociedad brindándoles apoyo en la reeducación desde los centros penitenciarios dotándoles de bibliotecas para la fomentación de la lectura como requisito mínimo de la educación. (p. 172), también Enjuanes y Morata (2019) refieren que la importancia de desarrollar actividades que fomenten la educación en los centros penitenciarios, donde se pueda influir mucho en mejorar la política de la reinserción social con una decidida intervención, el desarrollo de ambientes grupales y la transformación individual, permitirán potenciar sus competencias y consolidar procesos transitorios hacia la libertad, mientras se disminuye la discriminación social. (p. 12), del mismo modo Ordoñez (2016) manifiesta que trabajar proyectos de resocialización que permitan que la persona pueda desarrollar un plan de vida, así poder aprender y participar en labores internas de la prisión, en pocas palabras generar que la estancia en la prisión sea beneficiosa para los reos. (p. 22).

\section{Tabla 3}

Los programas de reinserción social vienen impulsando el crearoportunidades laborales

\begin{tabular}{cccc}
\hline Descripción & $\mathbf{f i}$ & \% \\
\hline & D & 11 & 11.00 \\
& NO & 34 & 34.00 \\
& A & 24 & 24.00 \\
& TA & 31 & 31.00 \\
\hline Total & & $\mathbf{1 0 0}$ & $\mathbf{1 0 0 . 0 0}$ \\
\hline
\end{tabular}

Fuente: El Autor

De acuerdo con los datos obtenidos de la Tabla 3, sobre si Los programas de reinserción social vienen impulsando el crear oportunidades laborales, al respecto un $11 \%$ solo refieren estar en Desacuerdo con tal afirmación, asimismo un 34 \% no tiene muy en claro tal situación y prefiere No Opinar, mientras que un $24 \%$ ante dicha afirmación refieren estar de Acuerdo y finalmente el $31 \%$ están Totalmente de Acuerdo con lo manifestado. Estos resultados los corroboramos con lo expuesto por Cacuango, Cadena y Carrillo (2020) quienes aseguran que la aceptación de los ex reclusos por la sociedad, se manifiesta, en que existe un rechazo generalizado por parte de los ciudadanos y de 
gerentes de negocios, incluso este rechazo es percibido por los ex reclusos ante los miembros de la sociedad. Se pretende que estas personas que reingresan a la sociedad tengan las mismas oportunidades que los demás, después de vivir su proceso penal y cumplir su condena. (p. 643).

\section{Tabla 4}

La incorporación reclusorio no está haciendo distinciones sobre la clasificación de internos por tipo de condena

\begin{tabular}{|c|c|c|}
\hline Descripción & $\mathbf{f i}$ & $\%$ \\
\hline $\mathrm{D}$ & 13 & 13.00 \\
\hline NO & 24 & 24.00 \\
\hline A & 31 & 31.00 \\
\hline TA & 32 & 32.00 \\
\hline Total & 100 & 100.00 \\
\hline
\end{tabular}

Fuente: El Autor

De acuerdo con los datos obtenidos de la Tabla 4, sobre si La incorporación reclusorio no está haciendo distinciones sobre la clasificación de internos por tipo de condena, al respecto un $13 \%$ solo refieren estar en Desacuerdo con tal afirmación, asimismo un 24 $\%$ no tiene muy en claro tal situación y prefiere No Opinar, mientras que un $31 \%$ ante dicha afirmación refieren estar de Acuerdo y finalmente el $32 \%$ están Totalmente de Acuerdo con lo manifestado. Esto se compara con lo expuesto por Monteverde, Castro y Saavedra (2018) quienes dicen en Chile se aplicó un proceso que vario por la involucración de intelectuales quienes figuraron a la hora de realizar críticas y recomendaciones para la aplicación de estos modelos extranjeros. Esta conducta fue resocializada con el objetivo de rebajar la tendencia de trasgresiones y concretar un modelo ordenado en las prisiones chilenas. (p. 90), asimismo Bové (2016) puntualiza que característicamente a la mitad de un problema institucional que trata de reformar el sistema penitenciario que atraviesa Uruguay, la solución sería una reforma que busca esquemas netamente punitivos, para asemejar a ejercicios de derechos humanos, es fundamental entender que no todo lo que se distancia de la mano dura es efectivo. La educación, en circunstancias precarias combate a diario con diferentes obstáculos. Los desarrollos se vuelven incuestionables, sujetos a la educación y a sistemas de castigar y premiar, estos sistemas no solo delimitan los sentidos educativos, sino que somete al ejercicio del derecho a incurrir al servicio de la 
educación con un fin disciplinal sobre el sistema carcelario (pp. 12-13).

\section{CONCLUSIÓN}

1) La presente investigación concluye con una propuesta sobre la implementación de un Programa de Reinserción Social para fortalecer el Modelo de Gestión Penitenciaria en las personas privadas de libertad en la ciudad de Guayaquil, Ecuador. 2020, el mismo que contiene elementos importantes en su diseño y que contribuirá a brindar una solución a la problemática de los modelos de gestión penitenciaria.

2) Se identificaron elementos trascendentes que se están manifestando e la dinámica de la gestión penitenciaria, donde se aprecian el hacinamiento de reclusos a causa de las variadas sentencias que se emiten, como acción sancionadora de parte de la justicia, la necesidad de brindarles soporte psicológico, brindarle capacitaciones educativas con el fin de rehabilitarlos, asimismo, generar la resocialización en el ámbito familiar, social y laboral.

3) Se analizaron factores que tuvieron una mayor prevalencia mostrándose a la incorporación permanente de reclusos como consecuencia del incremento de la criminalidad, la falta de preparación en actividades laborales que permitan tener una cultura del buen vivir, en la que la educación juega un rol muy importante.

4) Se elaboró un programa basándonos en el ferviente deseo de generar mejores condiciones de vida carcelaria dentro del marco de la gestión penitenciaria, una gestión que debe velar por el individuo desde su ingreso hasta su egreso, pero evidenciando que al egresar de ese centro penitenciario, el individuo sale con una preparación psicológica que le ayude a enrumbar su vida en el sentido y el marco de las leyes.

5) Los resultados del juicio de expertos demuestran la imperante necesidad de aplicar este programa, programa que se revisó y evaluó por especialistas, profesionales del ámbito de la gestión pública y de las leyes, análisis que arrojaron un Coeficiente de validez de contenido es 0.908 valor que recomienda la pertinencia de implementar y aplicar el programa.

\section{REFERENCIAS BIBLIOGRAFICAS}

Bové, M. (2016). Sentidos de la educación en una cárcel de mujeres: entre el tratamiento penitenciario, los modelos punitivos y de garantización de derechos. Revista Fermentario,

1(10). 
http://fermentario.fhuce.edu.uy/index.php/fermentario/article/view/226

Cacuango, G., Cadena, J., y Carrillo, M. (2020). Discernimiento E Inferencia De La Reinserción Social en Ecuador, Basada en Conjuntos De Números De 2-Tuplas. Investigación Operacional, 41(5), 637-646. https://rev-invope.pantheonsorbonne.fr/sites/default/files/inline-files/41520-05.pdf

Cadena, M. (2018). “Condiciones Ambientales del Internamiento Penitenciario y las Sentencias Elevadas, y su influencia en la Personalidad y Comportamiento, de las Personas Privadas de Libertad del Centro de Privación de Libertad de Personas Adultas en Conflicto con la Ley de Loja y Zamora Chinchipe, 20172018. Universidad Nacional de Loja. https://dspace.unl.edu.ec/jspui/bitstream/123456789/21368/1/CondicionesAmbientales-internamiento-penitenciario-sentencias-elevas-su-influencia- enla-personalidad-y-comportamieto.pdf

Dammer, L. (2018). La importancia de la rehabilitación: ¿qué funciona?. https://justicetrends.press/es/la-importancia-de-la-rehabilitacion-que- funciona/

De León Romero, L., Armijos, G., \& Rojas, R. (2021). Higher education programs in prisons. [Programas de formación universitaria en centros penitenciarios] Andamios, 18(45), 487-509. https://doi.org/10.29092/uacm.v18i45.827

Días, R., García, M., y Torres, J. (2021). Reinserción Social en el ámbito Laboral.

Universidad Nacional de La Pampa. pp. 1-48. https://repo.unlpam.edu.ar/handle/unlpam/7041

Domínguez Reyes, B., y Domínguez Cortés, S. M. (2020). Experiencia de la Clínica de Labio y Paladar Hendido del Hospital General de Tlaxcala: logros para la reinserción social completa de los pacientes tratados. Cirugía Plástica IberoLatinoamericana, 46(1), 107-112.

Durán, M., y Prado, G. (2020). Recomendaciones y propuestas para una reforma penitenciaria. Apuntes para su sistematización y delimitación. Revista de derecho (Valparaíso), (54), 151-181. https://dx.doi.org/10.4067/S071868512020005000104

Enjuanes, J., y Morata, T. (2019). Modelos penitenciarios educativos como base del éxito en la reinserción social de las personas privadas de libertad.

Boletín Criminológico, 25(2019). pp. 1-15. https://doi.org/10.24310/Boletin- 
criminologico.2019.v25i2019.7131

Enjuanes, J., y Morata, T. (2019). Modelos penitenciarios educativos como base del éxito en la reinserción social de las personas privadas de libertad.

Boletín Criminológico, (187), 1-15. https://revistas.uma.es/index.php/boletincriminologico/article/view/7131

Fornons, D., \& Barcelona, G. (2008). La práctica deportiva en la prisión: rehabilitación o evasión. Actualidad en el deporte: investigación y aplicación, 215-227 https://core.ac.uk/download/pdf/11500441.pdf

García, M. (2021). Conoce las técnicas de capacitación más efectivas para guiar a tu equipo. https://www.crehana.com/pe/blog/empresas/tecnicas-de- capacitacionmas-efectivas/

Gilardi, A. (2020). Del Juego a la Transgresión y de la Masculinidad a las Emociones. El Caso de Tona: Un Joven en Proceso de Reinserción Social en la Ciudad de México. Trayectorias, 22(50), 29-54. https://dialnet.unirioja.es/servlet/articulo?codigo $=7264214$

Guerrero, B. y Campaña, D. (2015). La Rehabilitación Social y el nuevo modelo de gestión del sistema penitenciario aplicables a los CRS de Guayaquil y CRS de Cotopaxi en el año 2015. Universidad Central Del Ecuador. pp. 1-

110. http://www.dspace.uce.edu.ec/handle/25000/5907

Gutierrez, E. (2019). Prevención general de las penas a través de los medios de comunicación. https://hayderecho.expansion.com/2019/09/04/prevenciongeneral-de-las-penas-a-traves-de-los-medios-de-comunicacion/

Gutierrez, E. (2019). Prevención general de las penas a través de los medios de comunicación. https://hayderecho.expansion.com/2019/09/04/prevenciongeneral-de-las-penas-a-traves-de-los-medios-de-comunicacion/

Gutiérrez, S. y Morales, M. (2019). Efectividad de la Rehabilitación Psicosocial en el funcionamiento y Reinserción Social en pacientes con Trastornos Mentales Graves. Universidad Norbert Wiener. pp. 1-42. http://repositorio.uwiener.edu.pe/handle/123456789/3074

Hernández, M. (2019). La reinserción social y el principio de proporcionalidad. Ciencia Jurídica, $\quad 8(16), \quad 49-68$. doi: https://doi.org/10.15174/cj.v8i16.312http://www.scielo.org.co/pdf/hpsal/v22n2/ 
0121-7577-hpsal-22-02-00099.pdfhttps://doi.org/10.5232/ricyde2020.06204 https://scielo.isciii.es/scielo.php?script=sci_abstract\&pid=S037678922020000100015

Jativa, M., y Alcivar, D. (2020). "Ineficacia del Sistema de Reinserción Social y Económica de Personas que han cumplido la pena por el delito de robo en la ciudad de Guayaquil parroquia Ximena. Universidad De Guayaquil. pp. 1-

82. http://repositorio.ug.edu.ec/handle/redug/50726

Jiménez, N. (2017). Resocialization as end of sentence - A frustration with the colombian penitentiary and prison system. [La resocialisation comme fin de peine - Une frustration dans le système pénitentiaire et à l'univers carcéral en Colombie; A ressocialização como final da pena - Uma frustração no sistema penitenciário e prisional Colombiano; La resocialización como fin de la pena Una frustración en el sistema penitenciario y carcelario Colombiano] Caderno CRH, 30(81), 539-559. https://doi.org/10.1590/S0103-49792017000300010

Kaufman, N. (2015). Prisoner incorporation: The work of the state and nongovernmental organizations. Theoretical Criminology, 19(4), 534-553. https://doi.org/10.1177/1362480614567172

Lapa, A. (2018). Gestión post penitenciaria y reeducación de los internos egresados de los establecimientos penitenciarios de la región Lima del Instituto Nacional Penitenciario - 2017. Universidad Cesar Vallejo. pp. 1-

107. https://repositorio.ucv.edu.pe/handle/20.500.12692/12658

Lavrentieva, M., Tchinaryan, E., Kuchenin, E., \& Turkin, M. (2020). Prevention of juvenile delinquency: social and legal aspects. EurAsian Journal of Biosciences, 14(2), 5515-5521. https://bit.ly/3dvG4lm

LLamoca, A. (2018). Gestión por Resultados del Tratamiento Penitenciario Lima 2018. Universidad Cesar $\quad$ Vallejo. $\quad$ pp. https://repositorio.ucv.edu.pe/handle/20.500.12692/21202

Machado, M., y Hernández, E. (2020). Rehabilitación y reinserción social: una quimera para los privados de libertad. Amauta “Todo Lo Humano Es Nuestro,"18(36), 27-42. https://core.ac.uk/download/pdf/288220143.pdf

Magnusson, R., McGrady, B., Gostin, L., Patterson, D., \& Taleb, H. (2019). Legal capacities required for prevention and control of noncommunicable diseases. 
Bulletin of the World Health Organization, 97(2), 108-117. https://doi.org/10.2471/BLT.18.213777

Maluche Sánchez, A., y Vilma Florisa Velásquez G. (2017). Efecto Programa De Apoyo Social: Percepción De Cuidadoras Familiares De Personas Mayores Afrocolombianas en Guapi, Cauca. Hacia La Promoción de La Salud, 22(2), 99110.

Martín-González, N., Martínez-Merino, N., Usabiaga-Arruabarrena, O., y MartosGarcía, D. (2020). Los significados que una madre presa y drogodependiente asigna a las actividades físico- deportivas: sus relaciones con la reinserción y la terapia. / The meanings that an inmate, mother and drug addict assigns to physical activities and sports: their relationship with rehabilitation and therapy. RICYDE. Revista Internacional de Ciencias Del Deporte, 16(62), 381-395.

Méndez, A. (2020). Modificación del artículo 27 Fracción IV de la ley nacional de ejecución penal, referente a la Reinserción Social. Benemérita universidad $\begin{array}{lllll}\text { Autónoma de } & \text { Puebla. } & \text { Pp. } & \text { 1-87. }\end{array}$ https://repositorioinstitucional.buap.mx/handle/20.500.12371/12477

Mendieta, L., Molina, B., y Huertas, O. (2020). Sistema progresivo penitenciario en Colombia: tratamiento y resocialización. IUSTA, (53), 15-44. https://doi.org/10.15332/25005286.6270

Menéndez, C. \& García, E. (2018). Características Predictoras de éxito en la Reinserción Social de personas drogodependientes. Universidad de Oviedo. https://dialnet.unirioja.es/servlet/articulo?codigo $=6621675$

Ministerio de Justicia (2018). ¿Cómo entendemos la Reinserción Social?. https://www.reinsercionsocial.gob.cl/que-es-la-reinsercion/

Ministerio de Justicia (2018). ¿Cómo entendemos la Reinserción Social?. https://www.reinsercionsocial.gob.cl/que-es-la-reinsercion/ 
Ministerio de Justicia (2018). ¿Cómo entendemos la Reinserción Social?. https://www.reinsercionsocial.gob.cl/que-es-la-reinsercion/

Montejano Torres, L., Galán Jiménez, J. S., y De la Rosa Rodríguez, P. I. (2019).

Reinserción social de adolescentes en conflicto con la ley. Un estudio conceptual. Estudios Socio-jurídicos, 22(1), 233-262. Doi: http://dx.doi.org/10.12804/revistas.urosario.edu.co/sociojuridicos/a.7606

Montero, E. (2018). La reeducación y la reinserción social en prisión: El tratamiento en el medio penitenciario español. Revista De Estudios Socioeducativos. ReSed, (7), 227-249. Recuperado a partir de https://revistas.uca.es/index.php/ReSed/article/view/4421

Monteverde Sánchez, A., Castro Valdebenito, H. J., y Saavedra Ávila, J. (2018).

Modelos, tendencias y cotidianidades enlos inicios de la Cárcel Penitenciaria de Santiago de Chile. 1843-1860. Diálogos Revista Electrónica de Historia, 19(1), 69-101. https://www.scielo.sa.cr/scielo.php?script=sci_arttext\&pid=S1409469X2018000100069

Monteverde, A., \& Estay, J. (2013). El sistema carcelario en Valparaíso 1836-1842 los carros y presidios ambulantes según fuentes documentales. Diálogos Revista Electrónica de Historia, 14(2), 145-165. http://www.scielo.sa.cr/scielo.php?script=sci_arttext\&pid=S1409469X2013000200005\&lng=en\&tlng=es.

Mora, D. (2003). Aplicaciones del régimen progresivo: Ubicación poblacional carcelaria y clasificación de los Centros de Rehabilitación Social y de los internos o presos de acuerdo a las nuevas Normas del Código de Ejecución de Penas y Rehabilitación Social y su reglamento y en la Constitución Política del Ecuador (Master's thesis, Quito, Ecuador). http://repositorio.iaen.edu.ec/handle/24000/245

Morales, A. (2019). Repositorio Institucional de la Universidad Politécnica Salesiana: Diseño de una herramienta de aprendizaje con realidad aumentada aplicada a la capacitación técnica. Repositorio Institucional de la Universidad Politécnica Salesiana. P. 1-54. https://dspace.ups.edu.ec/handle/123456789/17215

Morales, J. (2016). Gestión Penitenciaria y Reinserción Laboral en la Población Extramuros, en el Instituto Nacional Penitenciario - 2014. 
https://repositorio.ucv.edu.pe/bitstream/handle/20.500.12692/9115/Morales

_CJR.pdf? sequence=1\&isAllowed $=\mathrm{y}$

Morales, J. (2016). Gestión Penitenciaria y Reinserción Laboral en la Población Extramuros, en el Instituto Nacional Penitenciario - 2014. https://repositorio.ucv.edu.pe/bitstream/handle/20.500.12692/9115/Morales_CJ R.pdf? sequence $=1 \&$ isAllowed $=y$

Morales, J. (2018). Gestión Penitenciaria y Reinserción Laboral en la Población Extramuros, en el Instituto Nacional Penitenciario - 2014. Universidad Cesar Vallejo. pp. 1-128. https://repositorio.ucv.edu.pe/handle/20.500.12692/9115

Munizaga, A., y Sanhueza, G. (2017). Una revisión del modelo carcelario escandinavo con notas para Chile. TS Cuadernos de Trabajo Social, (16), 99-117.

http://www.tscuadernosdetrabajosocial.cl/index.php/TS/article/view/141

Narvaéz, J., y Shive, E. (2015). Implementación de una unidad de inteligencia penitenciaria que apoye el nuevo modelo de gestión penitenciaria en el Ecuador. Universidad San Francisco de Quito, pp. 1-64. https://repositorio.usfq.edu.ec/handle/23000/4545

Nestar, M. (2020). La pena de prisión y los modelos penitenciarios.Universidad de Valladolid.1-72. https://uvadoc.uva.es/handle/10324/46999

Novello, V. (2019). Naturaleza Jurídica de la Reinserción Social en el Sistema Penitenciario de adultos: ¿Un derecho o un beneficio?. Universidad De Chile. pp. 1-96. http://repositorio.uchile.cl/handle/2250/170567

Ochoa-García, J., y Martínez-Camacho, H. (2020). La función de la biblioteca penitenciaria en la reinserción social de los internos del Complejo Penitenciario Puente Grande, Jalisco, México. Informacion, Cultura y Sociedad, 43, 161-176. https://doi.org/10.34096/ics.i43.8063

Ordoñez, K. (2016). Impacto de los programas de resocialización en la reinserción social de la población reclusa. Universidad del Rosario. pp. 1-59. https://repository.urosario.edu.co/handle/10336/12840

Orjuela, C. (2020). Los beneficios de la reincorporación del Juez de Ejecución penal al sistema penitenciario del Perú. Pontificia Universidad Católica del Perú. Pp. 137. http://tesis.pucp.edu.pe/repositorio/handle/20.500.12404/16264

Pastor, E., y Torres, M. (2017). El sistema penitenciario y las personas privadas de 
libertad en España desde una perspectiva internacional. Política criminal, 12(23), 124-150. https://dx.doi.org/10.4067/S0718-33992017000100005

Pérez, J., \& Benito, S. (2020). University student's employment and labor insertion. [Empleo de los estudiantes universitarios y su inserción laboral] Revista De Educacion, 2020(390), 31-55. https://doi.org/10.4438/1988- 592X-RE-2020$390-464$

Peréz, J., y Merino, M. (2014). REINSERCIÓN SOCIAL. https://definicion.de/reinsercion-social/

Posada, M. (2016). Fines de la pena y derecho a la Reinserción Social en el Sistema $\begin{array}{llll}\text { Constitucional. Universidad } & \text { EAFIT. }\end{array}$ https://repository.eafit.edu.co/bitstream/handle/10784/11736/PosadaPuerta _MariaFernanda_2017.pdf?sequence=2

Preciado, V. (2020). Educación o resocialización: Problemática abordada desde la administración penitenciaria en Colombia. Utopia y Praxis Latinoamericana, 25, 139-153. https://doi.org/10.5281/zenodo.3907061

Puga, J. (2020). Programa de reconversión laboral para la inserción del personal militar en retiro al sector civil. Revista Científica General José María Córdova, 18(30), 439-458. http://dx.doi.org/10.21830/19006586.580

Rambal-Simanca, M., \& Acuña-Sauriht, L. (2021). Análisis De La Investigación “Imaginarios Sociales Del Desarrollo Humano, Subyacentes en Las Políticas De Resocialización De Mujeres Profesionales Internas, en El Centro Penitenciario Villa Cristina De La Ciudad De Armenia, Quindío, Colombia” Realizada Por Quiceno, J; Morales, L \& Cuellar, L, 2015. Journal of Research of the University of Quindio, 33(1), 126-131. https://doi.org/10.33975/riuq.vol33n1.321

Ramírez, B. y Pérez, M. (2017). Alcances y límites del Sistema de Reinserción Social en México. pp. 1-20. https://www.derechoycambiosocial.com/revista048/alcances_y_limites_del_ sistema_de_reinsercion.pdf

Requejo, L. (2017). Análisis de la Política Penitenciaria Nacional respecto de la rehabilitación de los presos primarios del penal de San Pedro-2016. https://hdl.handle.net/20.500.12692/18213

Romero, J., Cepeda, J., Quinn, P., \& Underwood, S. (2018). Prisoner rehabilitation 
through animal-assisted activities in argentina: The huellas de esperanza prison dog programme. [Resocialización de personas privadas de libertad a través de actividades con animales en Argentina: el programa «Huellas de Esperanza»] Revue Scientifique Et Technique (International Office of Epizootics), 37(1), 171180. https://doi.org/10.20506/rst.37.1.2750

Salinero, S., Morales, A., \& Castro, Á.. (2017). Análisis comparado y crítico de las alternativas a las penas privativas de libertad. La experiencia española, inglesa y alemana. . Política criminal, 12(24), 786-864. https://dx.doi.org/10.4067/S071833992017000200786

Salinero, S., y Fábrega, J. (2020). ¿Contribuye el aumento del catálogo de penas alternativas y el resto de modificaciones legales a cambiar el perfil de la población condenada en el sistema abierto? Evidencia para ChileRevista Criminalidad, 62(2): 181-198. http://www.scielo.org.co/pdf/crim/v62n2/17943108-crim-62-02-181.pdf

Sánchez-Gil, L., y Herrero, F. (2020). LOS CENTROS PENITENCIARIOS ESPAÑOLES COMO ESPACIOS DE RADICALIZACIÓN YIHADISTA.

Revista De Derecho Penal y Criminología, (23), 249-277. https://doi.org/10.5944/rdpc.23.2020.27431

SANTIBÁÑEZ-ORELLANA, J. (2014). Consecuencias Jurídico Penales en Contra De Las Personas Jurídicas en Colombia Y Políticas De Prevención Del Delito en Las Empresas: Una Mirada Al Modelo Chileno. Díkaion, 23(2), 447-451. https://doi.org/10.5294/dika.2014.23.2.9

Solórzano-Soto. R., Márquez-Allauca, V. y Márquez-Allauca, K. (2017). Terapia cognitiva-conductual para rehabilitación- reinserción social del adicto y minimización de factores biopsicosociales. Dom. Cien., ISSN: 2477-8818. 3(2), pp. $752-769$ https://dialnet.unirioja.es/servlet/articulo?codigo $=6325886$

Valencia, O., Ojeda, N., \& Hernández, W. (2020). Prácticas para prevenir la corrupción en las entidades públicas y privadas. Revista Logos Ciencia \& Tecnología, 12(2), 131-146. Epub October 05, 2020. https://doi.org/10.22335/rlct.v12i2.1142

Vasquez-Trespalacios, E., Atehortua-Salazar, S., Arango-Isaza, D., Gallego, C., \& Gallón Villegas, L. (2020). Reincorporación laboral de mujeres con cáncer de mama: experiencia de un centro oncológico en la ciudad de Medellín. 
Archivos de Prevención de Riesgos Laborales, 23(3), 315-329. Epub 21 de septiembre de 2020. https://dx.doi.org/10.12961/aprl.2020.23.03.02

Vidal, F. (2015). 'La entrada está en tus manos, la salida en las de Dios'. La religión como medio de rehabilitación de los presos en la cárcel de Belén y la penitenciaría de Lecumberri (1874-1900). Revista de Historia de las Prisiones, 2 . https://www.revistadeprisiones.com/wpcontent/uploads/2016/06/8_Sig\%C3\%BCenza.pdf.

Vilela, J. (2019). Características de organización espacial en base al desarrollo de las actividades de los bomberos, aplicados al diseño de una estación y centro de capacitación técnica para los bomberos voluntarios, Celendín 2019 (Tesis de licenciatura). Repositorio de la Universidad Privada del Norte. pp. 1-68. http://hdl.handle.net/11537/22411

Villa, K., Restrepo, A. y Cedillo, B. (2021). Una visión de la gestión penitenciaria en América Latina: a nueve meses del inicio de la pandemia del COVID-19. https://publications.iadb.org/publications/spanish/document/Una-vision-de- lagestion-penitenciaria-en-America-Latina-A-nueve-meses-del-inicio-de-lapandemia-del-COVID-19.pdf

Villalba, J. (2018). Training and Technical Assistance addressed to MSMEs. «Learned lessons». Revista Científica de la UCSA, 5(2), 3-5. https://doi.org/10.18004/ucsa/2409-8752/2018.005(02)003-005 